\title{
5-mehtyltetrahydrofolate rescues alcohol-induced neural crest cell migration abnormalities
}

\author{
Yu Shi ${ }^{1,2+}$, Jiejing $\mathrm{Li}^{3 \dagger}$, Chunjiang Chen ${ }^{2 \dagger}$, Manzi Gong ${ }^{1}$, Yuan Chen ${ }^{1}$, Youxue Liu ${ }^{2}$, Jie Chen ${ }^{2}$, Tingyu $\mathrm{Li}^{2^{*}}$ \\ and Weihong Song ${ }^{2,4^{*}}$
}

\begin{abstract}
Background: Alcohol is detrimental to early development. Fetal alcohol spectrum disorders (FASD) due to maternal alcohol abuse results in a series of developmental abnormalities including cranial facial dysmorphology, ocular anomalies, congenital heart defects, microcephaly and intellectual disabilities. Previous studies have been shown that ethanol exposure causes neural crest (NC) apoptosis and perturbation of neural crest migration. However, the underlying mechanism remains elusive. In this report we investigated the fetal effect of alcohol on the process of neural crest development in the Xenopus leavis.

Results: Pre-gastrulation exposure of 2-4\% alcohol induces apoptosis in Xenopus embryo whereas $1 \%$ alcohol specifically impairs neural crest migration without observing discernible apoptosis. Additionally, 1\% alcohol treatment considerably increased the phenotype of small head $(43.4 \% \pm 4.4 \%$, total embryo $n=234)$, and $1.5 \%$ and $2.0 \%$ dramatically augment the deformation to $81.2 \% \pm 6.5 \%(n=205)$ and $91.6 \% \pm 3.0 \%(n=235)$, respectively $(P<0.05)$. Significant accumulation of Homocysteine was caused by alcohol treatment in embryos and 5-mehtyltetrahydrofolate restores neural crest migration and alleviates homocysteine accumulation, resulting in inhibition of the alcohol-induced neurocristopathies.
\end{abstract}

Conclusions: Our study demonstrates that prenatal alcohol exposure causes neural crest cell migration abnormality and 5-mehtyltetrahydrofolate could be beneficial for treating FASD.

Keywords: Alcohol, 5-mehtyltetrahydrofolate, Neural crest, FASD, Xenopus

\section{Background}

Fetal alcohol spectrum disorders (FASD) results from maternal alcohol abuse and is featured by abnormal facial morphology, ocular anomalies, congenital heart defects, microcephaly and intellectual disability [1]. Dysregulation of neural crest $(\mathrm{NC})$ development has been contributing to the majority of malformations. Studies using numerous animal models reveal that alcohol can initiate neural crest cell apoptosis, and eventually develops FASD [2-4].

\footnotetext{
* Correspondence: tyli@vip.sina.com; weihong@mail.ubc.ca

${ }^{\dagger}$ Equal contributors

${ }^{2}$ Chongqing City Key Lab of Translational Medical Research in Cognitive Development and Learning and Memory Disorders, and Ministry of Education Key Lab of Child Development and Disorders, Children's Hospital of Chongqing Medical University, Chongqing 400014, China

${ }^{4}$ Townsend Family Laboratories, Department of Psychiatry, Brain Research Center, The University of British Columbia, 2255 Wesbrook Mall, Vancouver, BC V6T 1Z3, Canada

Full list of author information is available at the end of the article
}

$\mathrm{NC}$ cells are multipotent migratory cells originating at the ectoderm. After induction and specification, NC cells form many cell types ranging from the cranial bone, smooth muscle to peripheral and enteric neurons and glia [5-7]. NC development is tightly regulated by finely tuned gene regulatory network (GRN) and orchestrated hierarchically. NC induction is initialized at beginning of neurulation. Morphorgens such as Wnt, fibroblast growth factor (FGF), retinoic acid (RA) and bone morphogenetic protein (BMP) secreted from the paraxial mesoderm and epidermis regulates the expression of a group of transcription factors (Pax3, Zic1, Msx1) whereby defines the boarder of neural crest. Subsequently, these transcription factors stimulate the expression of the NC specification genes including FoxD3, Slug and Twist to determine the cell fate of NC $[8,9]$. Once NC induction is accomplished NC cells undergo the epithelial-mesenchymal transition (EMT) and eventually migrate along predetermined trajectories.

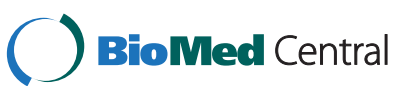

(C) 2014 Shi et al.; licensee BioMed Central Ltd. This is an Open Access article distributed under the terms of the Creative Commons Attribution License (http://creativecommons.org/licenses/by/4.0), which permits unrestricted use, distribution, and reproduction in any medium, provided the original work is properly credited. The Creative Commons Public Domain Dedication waiver (http://creativecommons.org/publicdomain/zero/1.0/) applies to the data made available in this article, unless otherwise stated. 
Given NC induction and migration occurs sequentially $[10,11]$, induction associated neurocristopathies can be identified to reflect migration abnormality. Alcohol appears to be able to affect $\mathrm{NC}$ development via either interfering $\mathrm{NC}$ cell migration or prompting cell apoptosis [12] that is indistinguishable from induction defects. Folate deficiency has long been known to be involved in NC defects. Abnormal folic acid level leads to dysfunction of NC migration $[11,13]$. The disturbance of folic acid metabolism causes craniofacial malformation [14,15] and heart defects [16]. Furthermore, studies showed that supplementation of folic acid successfully modulates alcohol-induced gene expression profiles and alleviates the developmental defects $[17,18]$. However, the exact mechanism underlying on what specific developmental phase folic acid affects remains elusive.

In this study using Xenopus model system we investigated whether alcohol directly affected migration. We found that pre-gastrulation exposure of 2-4\% alcohol induces developmentally non-specific apoptosis, whereas $1 \%$ alcohol specifically impairs neural crest migration without significant apoptosis, which consequently lead to neurocristopathies-like abnormalities. Furthermore, we showed that homocysteine, a molecular reported to affect neural crest migration [19], was elevated after alcohol exposure and 5-mehtyltetrahydrofolate (5MTHF) ameliorated endogenous homocysteine levels induced by alcohol exposure and dramatically rescued the impaired neural crest migration.

\section{Results and discussion}

\section{Alcohol induces neurocristopathies in Xenopus leavis}

To examine FASD in Xenopus, embryos are treated with 0-2\% alcohol starting from pre-gastrulation (stage 9) and washed at late neural plate stage (stage 16) and then cultured in modified Barth solution (MBS). Since cartilage architecture and pigment cell patterning are the most prominent developmental structure of NC derivatives, we examined malformations of cartilage and pigment cell development at stage 45. Alcohol treatment resulted in deformations of the pigment (Figure 1B; red arrow and Figure 1D) and the head structure (Figure 1B; red arrow head) compared to control group (Figure 1A; blue arrow and Figure 1C). Alcohol exposure also led to aberrant cranial cartilage formation with a shrunk dysplasic size and the small head (Figure 1E). In terms of small head phenotype, there is no significant different between $0.5 \%$ alcohol $(2.3 \% \pm 0.7 \%, \mathrm{n}=214)$ and control group $(2.3 \% \pm 1.5 \%, \mathrm{n}=190) \quad(\mathrm{P}>0.05)$. However, $1 \%$ alcohol significantly increased the phenotype of small head $(43.4 \% \pm 4.4 \%, \mathrm{n}=234)$, and $1.5 \%$ and $2.0 \%$ dramatically augment the deformation to $81.2 \% \pm 6.5 \%(n=205)$ and $91.6 \% \pm 3.0 \%(n=235)$ respectively $(P<0.01)$ (Figure $1 F)$. The results indicate that alcohol destructs neural crest normal development in a dosage dependent manner in Xenopus model system.

\section{Alcohol causes neither neural crest induction nor cell apoptosis at neural plate stage}

The development of NC has two functionally connected but developmentally separated phases that involve induction and migration. FASD could be the consequence of dysregulating of either induction or migration. Previous studies mainly focused on characterizing its impact on NC migration. Here we investigated how alcohol specifically affected NC induction. Xenopus embryos were incubated with $0.5 \%$ to $2 \%$ alcohol at the onset of gastrulation (stage 9) and collected at neural stages (stage 16). An in situ hybridization was performed using the border markers Pax3 and Zic1 as well as NC marker slug. The expression patterns of all three genes were not affected during the differentiation of the pre-migratory neural crest (Figure 2A and B). RT-PCR assays were performed to further confirm the results. Consistent with the in situ hybridization data, the expression levels of the border genes Msx1, Pax3 and Zic1 and neural crest marker gene Slug showed similar intensity to those in control embryos (Figure 2C). These results suggest that alcohol exposure does not affect NC induction.

Dysregulation of cell proliferation and apoptosis are implicated in alcohol-triggered neural crest deficiency $[12,20,21]$. However, results from different species are controversial and inconsistent [22]. We examined this issue in Xenopus model system using immunohistochemistry with phosphohistone $\mathrm{H} 3(\mathrm{pH} 3)$ and activated caspase-3 antibodies to detect cell proliferation and apoptosis, respectively. The Xenopus embryos were incubated in alcohol-MBS buffer at stage 9 until harvest time at stage 16 . Alcohol exposure at $2 \%$ did not change the number of $\mathrm{pH} 3$ positive cells in the $\mathrm{NC}$ region at stage 16 (Figure 2D pink cells inside white dot line box). The cell apoptosis was not increased at stage 16 until the alcohol concentration over $2 \%$ as shown by activatedcaspase 3 western blot assay (Figure 2E). Our data suggest that alcohol exposure $(<2 \%)$ does not affect cell proliferation nor apoptosis in Xenopus neural crest induction.

\section{Alcohol impairs NC cell migration}

Next we examined whether alcohol exposure affects NC cell migration. The embryos from stage 9 were treated with alcohol and washed at stage 16, and then cultured in MBS until tail bud stage. An in situ hybridization assay was performed to determine the expression of migrating marker genes of NC cells-Twist and Slug. Twist and Slug positive cells migrated ventrally in several streams into the hyoid and branchial arches of the Xenopus embryo. In control embryos, Twist (Figure 3A) and Slug (Figure 3F) were expressed in distally migrating NC cells. These 

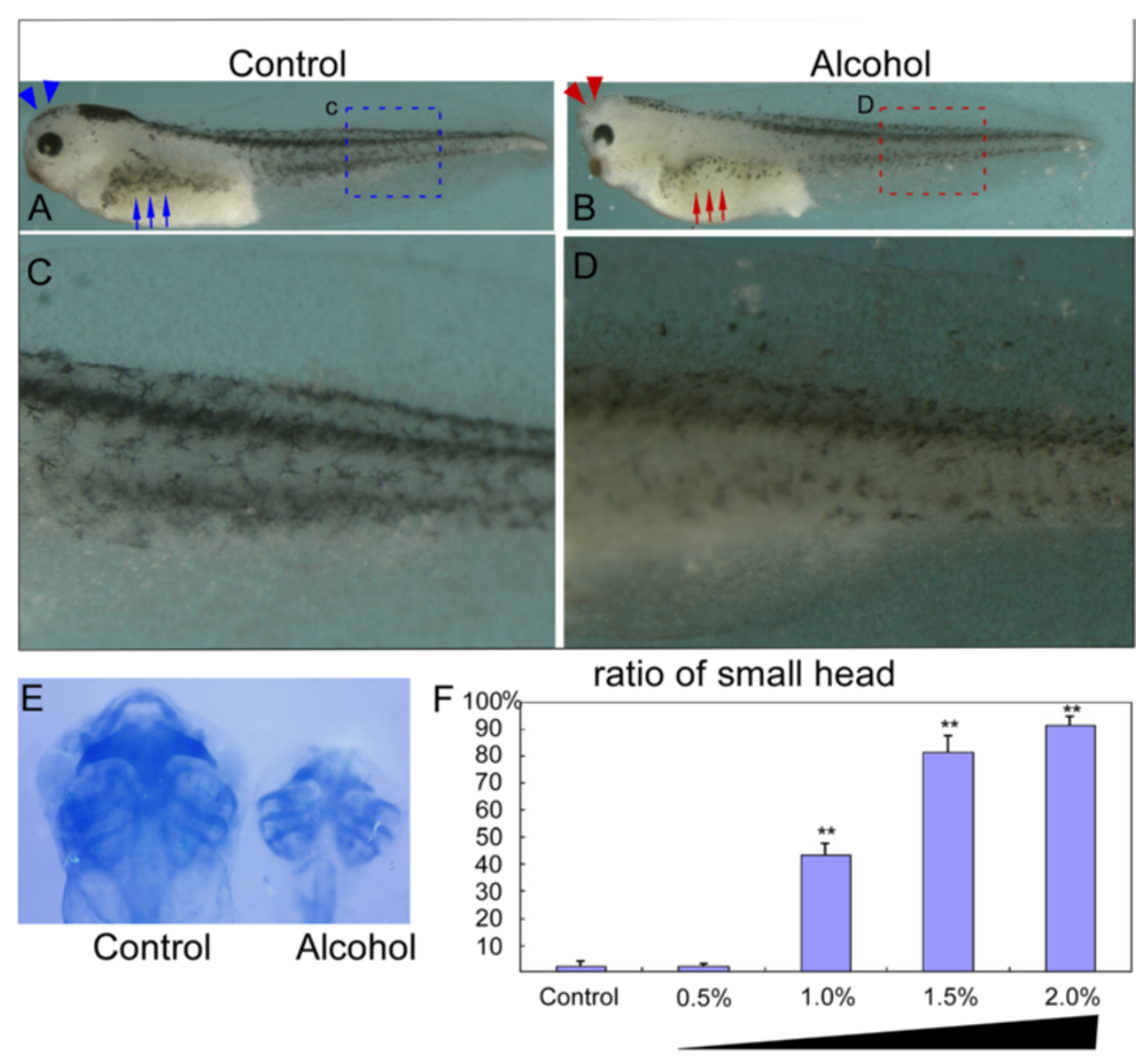

Alcohol

Figure 1 Alcohol exposure impaired pigment and cranial cartilage formation. $(A, C)$ Control embryos show normal amount and pattern of pigment cells. (B,D) Alcohol treated embryos develop less and punctate pigment cells. (E) Alcohol treated embryos at stage 45 exhibited truncated cranial cartilage. Compared to control embryos, both Meckel's and palatoquadrate were absent from treated embryos and the ceratohyal cartilages were markedly smaller. (F) The alcohol exposure deteriorates cartilage formation. Ratio of small to total heads exhibit no significant difference between control $(2.3 \% \pm 1.5 \%, n=190)$ and $0.5 \%$ alcohol condition $(2.3 \% \pm 0.7 \%, n=214)$. Additionally, $1 \%$ alcohol treatment considerably increased the phenotype of small head $(43.4 \% \pm 4.4 \%$, total embryo $n=234)$, and $1.5 \%$ and $2.0 \%$ dramatically augment the deformation to $81.2 \% \pm 6.5 \%(n=205)$ and $91.6 \% \pm 3.0 \%(n=235)$ respectively $(P<0.01)$. At each concentration, the experiments were repeated 3 times, and $n$ represents the total embryos of triplicate assay. The values represent means \pm SEM. $p<0.01$, by student T test.

markers were confined to much more dorsal regions of the embryo in alcohol exposure groups. This result suggest a decrease in the migration distance that the separated NC streams had moved. In addition, NC cell migration was impaired by alcohol exposure in a dosage dependent manner (Figure 3B-E, G-J). With 2\% alcohol treatment, NC cells failed to migrate ventrally into separate streams and were clustered and retained in a dorsal position (Figure 3E, J).

\section{MTHF rescues alcohol-induced neural crest migratory abnormality in Xenopus model}

Previous experiments have shown that alcohol consumption decreased the ability of folic acid uptake [23] and accumulated homocysteine in rodent model and human $[24,25]$. Furthermore, homocysteine has been found to block NC migration [19]. Interestingly, folic acid was found to reverse alcohol induced-teratogenesis in a mouse model $[17,18]$. In addition, our recent data indicated that folic acid was involved in NC migration [11]. The inhibition of NC migration by alcohol could be attributed to SAM-homocysteine dysregulation. To examine this issue, embryonic homocysteine levels were first evaluated under a $2 \%$ alcohol exposure from Xenopus development stage 9. At the NC pre-migratory time (stage 16), alcohol exposure did not significantly change the amount of endogenous homocysteine $(23.5 \pm 2.5 \mu \mathrm{M}$ in control and $23.0 \pm$ $2.3 \mu \mathrm{M}$ in alcohol, $\mathrm{P}>0.05)$. However, alcohol treatment clearly increased homocysteine levels at stage 27 when the $\mathrm{NC}$ cells underwent robust emigration $(25.5 \pm 3.7 \mu \mathrm{M}$ in control and 41.8 $\pm 6.3 \mu \mathrm{M}$ in alcohol, $\mathrm{P}<0.05$ ) (Figure 4A). Microinjection of 5MTHF, the most bioactive form of folic acid, attenuated alcohol-induced elevations of homocysteine levels $(30.5 \pm 2.3 \mu \mathrm{M})$ (Figure $4 \mathrm{~A}$ ). To determine 
A

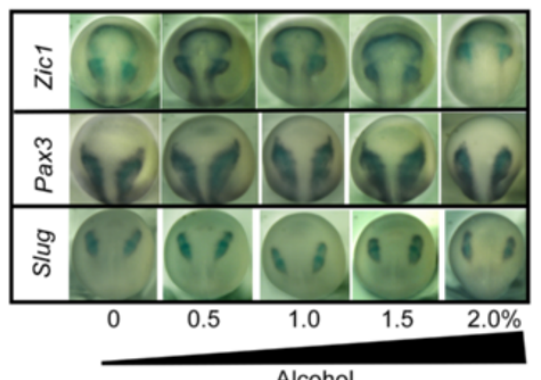

Alcohol

C

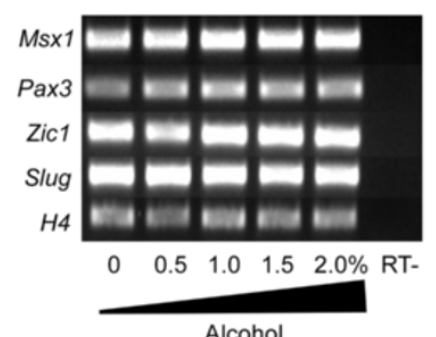

Alcohol

D

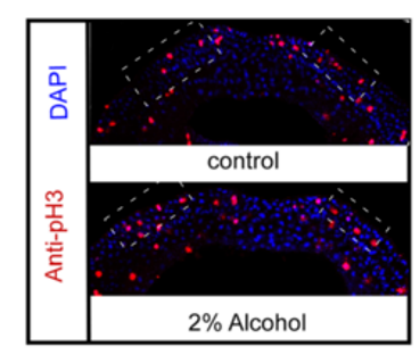

E
B
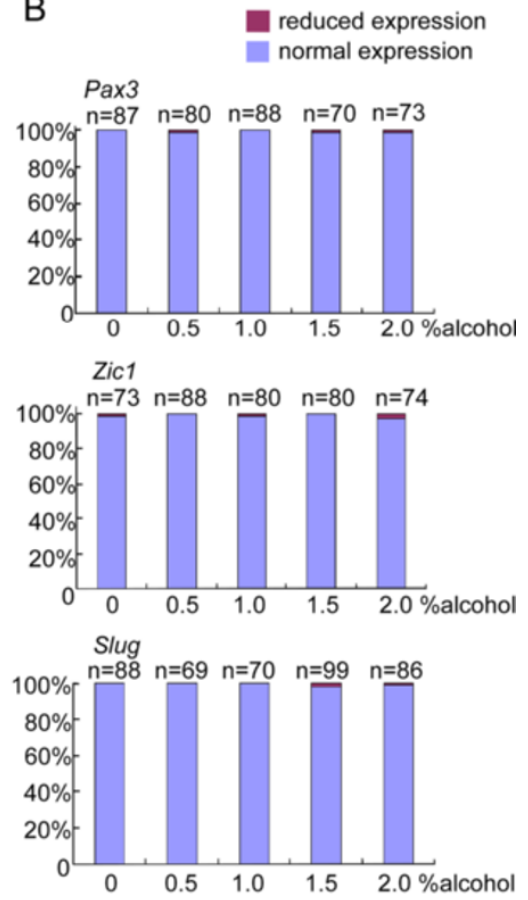

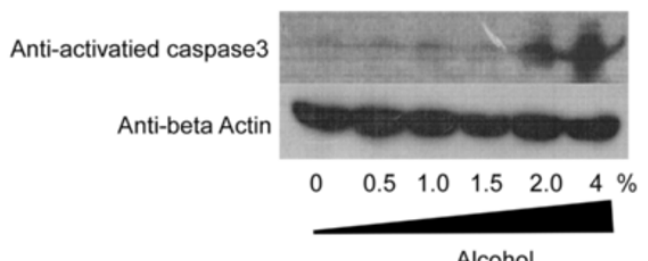

Alcohol

Figure 2 Exposure to lower concentration of alcohol had no significant toxicity to neural crest development. We harvested the embryos at stage 16 for Whole-mount In Situ Hybridization (WISH). (A) Both boarder determinator gene Pax3, Zicl and neural crest specification marker gene slug are kept intact. (B) Quantification of the effect of alcohol exposure on the expression of neural crest markers. (C) RT-PCR assays display expression of Msx1, Pax3, Zic1 and Slug are identical according to divergent alcohol treatment. (D,E) Alcohol less than $2 \%$ did not trigger abnormality of cell proliferation (labeled with pH3). Neural crest cell apoptosis was induced by $2 \%$ alcohol treatment as shown by activated caspase3. (normalized with beta actin).

whether 5MTHF alleviated the effect of alcohol on NC migration, the embryos were injected with 5MTHF at two cell stages prior to being immersed in the alcoholcontaining MBS buffer. The neural crest migration was analyzed with an in situ hybridization assay of Twist expression at the tadpole stage. Twist positive cells, absent in the most ventral region of branchial arch upon alcohol exposure (Figure 4B), were clearly present in most of the 5-MTHF injected embryos (Figure 4C). To further exclude that the absence of Twist expression was not due to any disabled differentiation processes (i.e. the inability of twist expression to further induce cellular differentiation) during the migration of the $\mathrm{NC}$ cells, embryos were first labeled by injection of a GFP mRNA into one blastomere at the two cell stage. The GFP-positive neural crest cells from the injected blastomere were dissected from the donor embryos and transplanted into the similar place of normal embryos at the neurula stage (Figure 4D). In MBS control groups $(n=18)$, these cells were found to migrate ventrally in three fluorescent streams (Figure 4E). However, alcohol incubation at the beginning of gastrulation to NC transplant time (stage 16) of donor embryos partially or completely impaired the ventral movement of the fluorescent streams (Figure 4F, G). Upon 0.5\% alcohol exposure, $12.5 \%$ fluorescent graft exhibited partial migration $(n=16)$. At $1 \%$ alcohol concentration, most of the grafts $(22 / 25)$ migrated partially and 2 grafts exhibit non-migration. The percentage of migration impairment was increased up to $62.5 \%$ partial migration, $37.5 \%$ non-migration $(\mathrm{n}=16)$ at $1.5 \%$ alcohol exposure. 


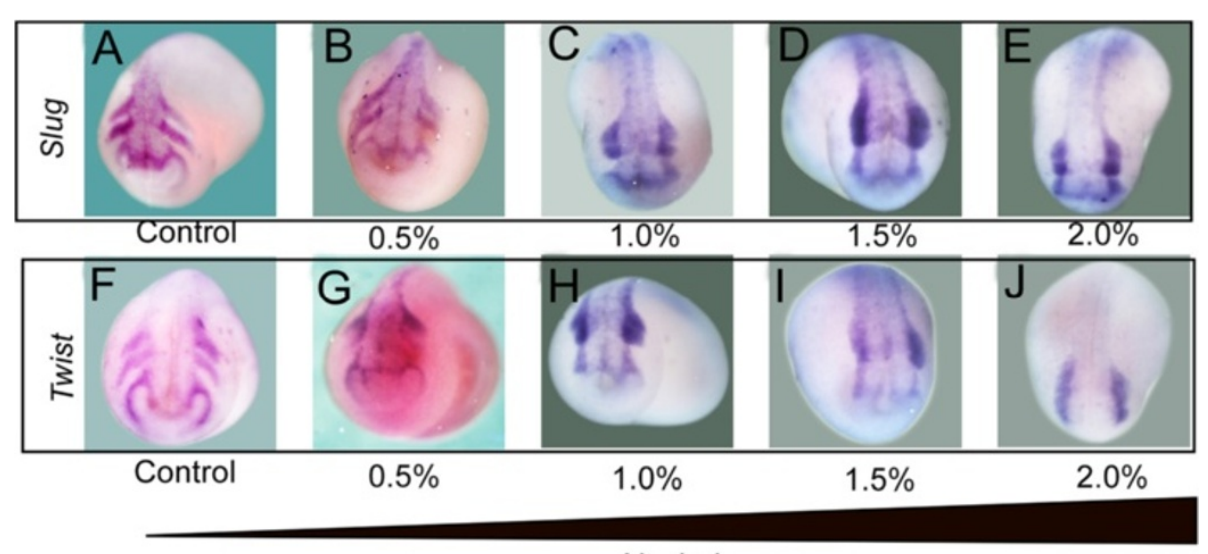

Alcohol

Figure 3 Alcohol exposure disrupts neural crest migration. (A) Both slug and twist1 in situ hybridization, performing along with gradient alcohol treatment, displays neural crest migration is accordingly blocked by the increasing concentration of alcohol. (B,G) indicates $0.5 \%$ alcohol treatment still allows fairly migration of neural crest versus control embryos (A,F). 1.0\% alcohol treatment deteriorate the egressing process of neural crest, however, there is perceivable migration occurring (C,H). Finally, both $1.5 \%$ and $2.0 \%$ appears completely freezing the migration $(\mathbf{D}, \mathbf{E}, \mathbf{I}, \mathbf{J})$.

$2 \%$ alcohol exposure resulted in $47.6 \%$ partial migration and $52.4 \%$ non-migration $(\mathrm{n}=21$ ) (Figure $4 \mathrm{H})$ and this migratory impairment was alleviated to $55.5 \%$ partial migration and $16.7 \%$ non-migration with $27.8 \%(n=18)$ showing normal migration when the donor embryos were injected with 5-MTHF at the 2 cell stage (Figure $4 \mathrm{H}$ ). These results suggested that alcohol affected folic acidhomocysteine metabolism and thereby disrupted the neural crest migration in Xenopus model.

FASD has been largely attributed to dysregulation of NC development via either disruption of migration or cell apoptosis. In this study we found that alcohol treatment at $2 \%$ triggers apoptosis in Xenopus embryos, leading to neural crest deformations. Interestingly, we found that both $1.5 \%$ and $1.0 \%$ specifically inhibited NC migration without detectable apoptosis. These results indicate that alcohol preferentially affects the initiation of $\mathrm{NC}$ migration. Moreover, we found that the process of $\mathrm{NC}$ induction was not affected at $1.5 \%$ and $1.0 \%$ of alcohol treatment, suggesting that alcohol specifically interferes with NC migration. Our study suggest that human FADS could be more related to defective NC migration than apoptosis since human blood alcohol concentration is usually less than $0.5 \%$.

FASD is a developmental disorder and the interaction between environment and gene plays a major role in the pathogenesis of FASD [26,27]. Our study showed that 5-MTHF could potentially prevent the alcohol-induced developmental abnormalities. 5-MTHF plays a critical role in nucleotide synthesis and methylation processes. 5-MTHF is important to recycle homocysteine and synthesize S-adenosylmethionine (SAM). SAM is the main methyl donor providing methyl residue for the most of biological methylation reactions [28]. Our previous work has indicated that modulating the metabolism of 5-MTHF affected the histone 3 lysine (H3K) methylation during neural crest development [11]. Furthermore, it has been reported that the H3K methylation preferentially takes place at Snail and Twist1 enhancer, consequently controlling neural crest migration by altering transcriptional accessibility [29]. Our study showed that alcohol resulted in accumulation of homocysteine at relatively late stage, indicating decreased level of 5-MTHF [30-32] during early neural crest development. Addition of 5-MTHF enables neural crest the capability of blocking the detrimental effects of alcohol on development. Clinical studies also showed significantly enhancement of serum homocysteine level upon alcohol consumption $[24,25]$. Homocysteine has been known as a risk factor for neurocristopathies [33-36]. Alcohol appears to perturb neural crest migration by obstructing 5-MTHF absorption and increasing homocysteine. These detrimental effect could result in the abnormalities in FASD. Thus, modulation of one carbon cycle might be beneficial to prevention of FASD.

During embryonic development, alcohol targets many types of cell and/or organ [37-41]. Importantly, our observations are consistent with previous studies that alcohol causes NC migratory defectives via promoting apoptosis. Meanwhile, our results further indicate that the dysregulation of one carbon cycle during NC migration could be a novel mechanism underlying FASD. This discovery shed a light on the therapeutic possibility of folic acid. Our experiments were specifically designed to investigate toxicity of alcohol on NC development. Mesoderm plays a center role from the initiation to the migration of $\mathrm{NC}$ development, and tightly regulates the formation of whole body plan. Thus, further study is warranted to examine the role of mesoderm in NC development and its effect on FASD pathogenesis. 


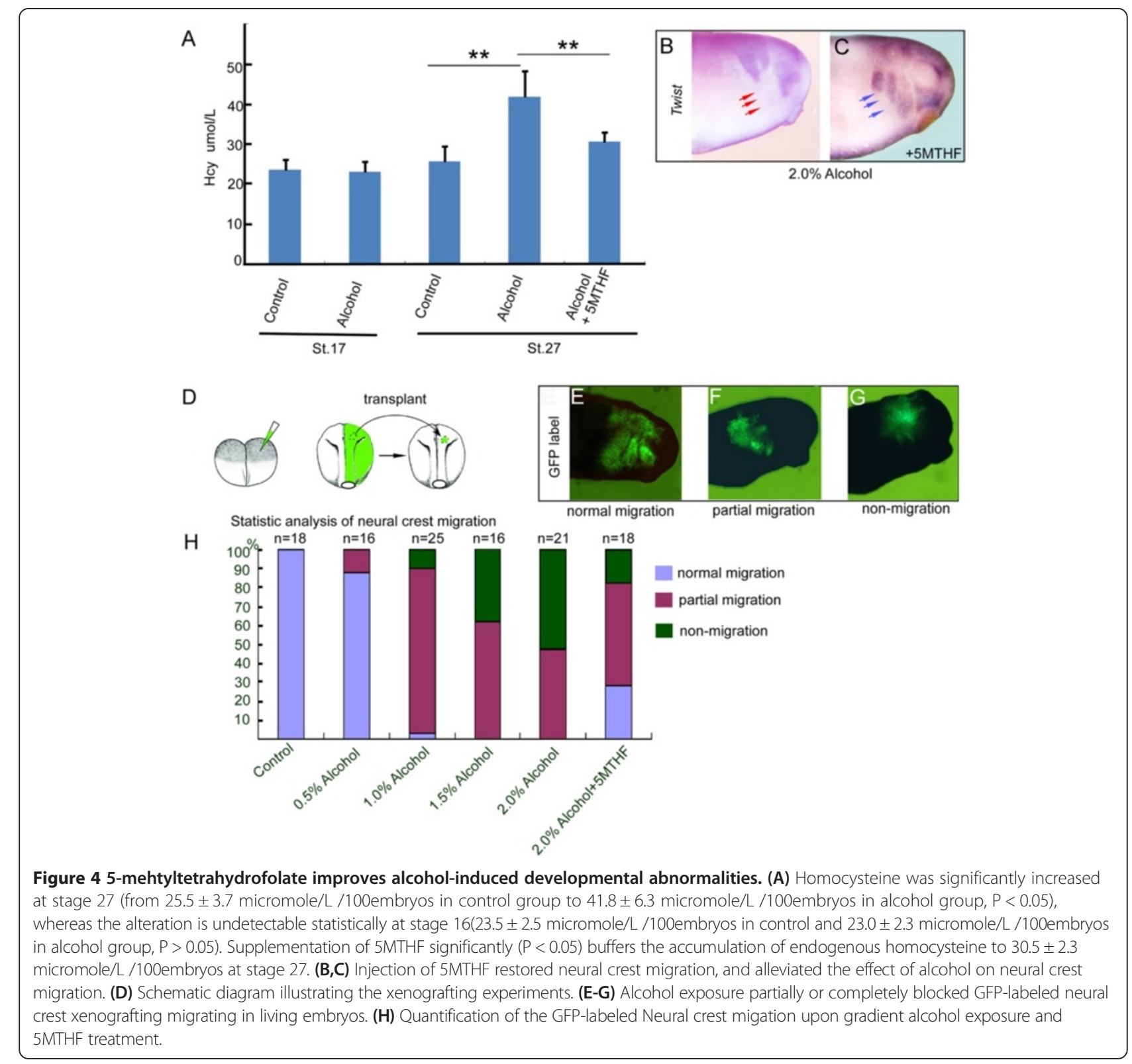

\section{Conclusions}

Our study demonstrate that prenatal alcohol exposure causes neural crest cell migration abnormality and 5mehtyltetrahydrofolate could be beneficial for treating fetal alcohol spectrum disorders.

\section{Methods}

\section{Xenopus experiments}

The experimental procedures for in vitro fertilization, manipulation and microinjection of the Xenopus embryos, as well as for the in situ hybridization of whole mount embryo were previously described [42]. The cartilage staining was carried out using Alcian blue 8GX according to protocol of Dr. Richard Harland's lab (http://tropicalis. berkeleyedu/home/gene_expression/cartilage-stain/alcian. html) as well as described [43] and the in situ probes for Zic1, Pax3 Slug and Twist gene expression were described previously [44]. Microinjection was performed using the PLI-1 Pico-injector (Harvard Apparatus) equipped with the MK-1 micromanipulator (Singer Instruments).

Reverse transcription - polymerase chain reaction (RT-PCR)

To test the effect of alcohol on neural crest induction, RT-PCR was carried out using whole embryos at the onset of gastrulation (stage9) until stage 16. H4 was used as a loading control. The primers used for PCR were: Pax3: 5'-CAGCCGAATTTTGAGGAGCAAAT-3' and 5'-GGG CAGGTCTGGTTCGGAG TC-3'; Snail2: 5' -TCC- CGC 
ACTGAAAATGCCACGATC -3' and 5' - CCGTCCTAAAGATGAAGGGTATCCTG -3'. The primers for Msx1 were used as described [11]. Please simply describe the procedure.

\section{Embryo immunohistochemistry and western blot}

Embryos were collected at stage 16 and fixed with paraformaldehyde. The frozen samples were sectioned in $10 \mu \mathrm{m}$ and the sections were stained with 1:200 Anti-phosphohistone H3 (Millipore 16-657) to detect cell proliferation and $300 \mathrm{nM}$ DAPI (Life Technologies D1306) for nuclear conterstain. Whole embryos at stage 16 were lysed with $1 \%$ triton-X100 in PBS containing a protease inhibitor cocktail (Roche) and the samples were subject to Western blot analysis with activated caspase3 antibody(Abcam 13847) to check cell apoptosis.

\section{Homocysteine measurement}

One hundred Xenopus embryos were collected at either stage 16 and 27 and homogenized in $500 \mu$ of lysis buffer [1\%triton-X100 in PBS containing a protease inhibitor cocktail (Roche)]. The samples were centrifuge at $12000 \mathrm{~g}$ for $10 \mathrm{~min}$ at $4^{\circ} \mathrm{C}$. After centrifugation, samples were separated into 3 phases; the lower sediment, intermediate water phase (which contained homocysteine) and upper lipid phase. The intermediate water phase was carefully collected without any lipid contamination. The homocysteine concentration was determined by an enzymatic method (produced by Zhongyuan Bio. Ltd China) on an automatic analyzer (Olympus AU 2700). The linear range of Hcy measurement is from $3 \mu \mathrm{M}$ to $50 \mu \mathrm{M}$.

\section{Statistical analysis}

All experiments were repeated at least 3 times, and the $P$ value were calculated with T-TEST.

\section{Competing interests}

The authors declare no competing financial interests.

\section{Authors' contributions}

YS, JL, CC, TL, and WS conceived and designed the experiments; YS, JL, and CC performed the experiments; YS, JL, YL, and WS analyzed the data; MG and $Y C$ contributed reagents/materials/analysis tools; YS and WS wrote the paper. All authors read and approved the final manuscript.

\section{Acknowledgements}

We would like to thank Dr. Bingyu Mao for the experimental reagents. This work was supported by the National Natural Science Foundation of China (NSFC) Grants 81102519 (to Y.S.), 81200878 (J.L.), 81161120498 (T.L.), China Postdoctoral Science Foundation funded project 2012 M511914 (Y.S.), Chongqing Science and Technology Committee Grant cstc2012jjA0147 (Y.S.), and Canadian Institutes of Health Research (CIHR) Operating Grant TAD-1 17948 (W.S). The funders had no role in the study design, data collection and analysis, decision to publish, or preparation of the manuscript.

\section{Author details}

${ }^{1}$ Department of Clinical laboratory, Chongqing, China. ${ }^{2}$ Chongqing City Key Lab of Translational Medical Research in Cognitive Development and Learning and Memory Disorders, and Ministry of Education Key Lab of Child Development and Disorders, Children's Hospital of Chongqing Medical
University, Chongqing 400014, China. ${ }^{3}$ Department of Biology, West Virginia University, Morgantown, WV, USA. ${ }^{4}$ Townsend Family Laboratories, Department of Psychiatry, Brain Research Center, The University of British Columbia, 2255 Wesbrook Mall, Vancouver, BC V6T 1Z3, Canada.

Received: 25 July 2014 Accepted: 29 August 2014

Published online: 16 September 2014

\section{References}

1. Khalil A, O'Brien P: Alcohol and pregnancy. Obstet Gynaecol Reprod Med 2010, 20(10):311-313.

2. Wang G, Bieberich E: Prenatal alcohol exposure triggers ceramide-induced apoptosis in neural crest-derived tissues concurrent with defective cranial development. Cell Death Dis 2010, 1(5):e46.

3. Cartwright MM, Smith SM: Increased Cell Death and Reduced Neural Crest Cell Numbers in Ethanol-Exposed Embryos: Partial Basis for the Fetal Alcohol Syndrome Phenotype. Alcohol Clin Exp Res 1995, 19(2):378-386.

4. Debelak KA, Smith SM: Avian genetic background modulates the neural crest apoptosis induced by ethanol exposure. Alcohol Clin Exp Res 2000, 24(3):307-314

5. Minoux M, Rijli FM: Molecular mechanisms of cranial neural crest cell migration and patterning in craniofacial development. Development 2010, 137(16):2605-2621.

6. Fröb F, Bremer M, Finzsch M, Kichko T, Reeh P, Tamm ER, Charnay P, Wegner M: Establishment of myelinating schwann cells and barrier integrity between central and peripheral nervous systems depend on Sox10. Glia 2012, 60(5):806-819.

7. Jain R, Engleka KA, Rentschler SL, Manderfield LJ, Li L, Yuan L, Epstein JA: Cardiac neural crest orchestrates remodeling and functional maturation of mouse semilunar valves. J Clin Invest 2011, 121(1):422-430.

8. Betancur P, Bronner-Fraser M, Sauka-Spengler T: Assembling neural crest regulatory circuits into a gene regulatory network. Annu Rev Cell Dev Biol 2010, 26:581-603.

9. Milet C, Monsoro-Burq AH: Neural crest induction at the neural plate border in vertebrates. Dev Biol 2012, 366(1):22-33.

10. Pegoraro C, Monsoro-Burq AH: Signaling and transcriptional regulation in neural crest specification and migration: lessons from xenopus embryos. Wiley Interdiscip Rev Dev Biol. 2013, 2(2):247-259.

11. Li J, Shi Y, Sun J, Zhang Y, Mao B: Xenopus reduced folate carrier regulates neural crest development epigenetically. PLOS ONE 2011, 6(11):e27198.

12. McCarthy N, Wetherill L, Lovely CB, Swartz ME, Foroud TM, Eberhart JK: Pdgfra protects against ethanol-induced craniofacial defects in a zebrafish model of FASD. Development 2013, 140(15):3254-3265.

13. Etchevers $\mathrm{H}$, Amiel J, Lyonnet S: Molecular bases of human neurocristopathies. Neural Crest Induction Differ 2006, 589:213-234.

14. Finnell RH, Greer KA, Barber RC, Piedrahita JA, Shaw GM, Lammer EJ: Neural tube and craniofacial defects with special emphasis on folate pathway genes. Crit Rev Oral Biol Med 1998, 9(1):38-53.

15. Prescott NJ, Malcolm S: Folate and the face: Evaluating the evidence for the influence of folate genes on craniofacial development. Cleft Palate-Craniofacial J 2002, 39(3):327-331.

16. Goldmuntz E, Woyciechowski S, Renstrom D, Lupo PJ, Mitchell LE: Variants of folate metabolism genes and the risk of conotruncal cardiac defects. Circulation Cardiovascular Genetics 2008, 1(2):126-132.

17. Wang LL, Zhang Z, Li Q, Yang R, Pei X, Xu Y, Wang J, Zhou SF, Li Y: Ethanol exposure induces differential microRNA and target gene expression and teratogenic effects which can be suppressed by folic acid supplementation. Hum Reprod 2009, 24(3):562-579.

18. $X u$ Y, Tang $Y, L i$ Y: Effect of folic acid on prenatal alcohol-induced modification of brain proteome in mice. Br J Nutr 2008, 99(3):455-461.

19. Brauer PR, Rosenquist TH: Effect of elevated homocysteine on cardiac neural crest migration in vitro. Dev Dyn 2002, 224(2):222-230.

20. Swartz ME, Wells MB, Griffin M, McCarthy N, Lovely CB, McGurk P, Rozacky J, Eberhart JK: A screen of zebrafish mutants identifies ethanol-sensitive genetic loci. Alcohol Clin Exp Res 2013, 38(3):694-703.

21. Boric K, Orio P, Viéville T, Whitlock K: Quantitative analysis of cell migration using optical flow. PLOS ONE 2013, 8(7):e69574.

22. Oyedele $\mathrm{OO}$, Kramer B: Nuanced but significant: How ethanol perturbs avian cranial neural crest cell actin cytoskeleton, migration and proliferation. Alcohol 2013, 47(5):417-426. 
23. Said HM, Mee L, Sekar VT, Ashokkumar B, Pandol SJ: Mechanism and regulation of folate uptake by pancreatic acinar cells: effect of chronic alcohol consumption. Am J Physiol Gastrointest Liver Physiol 2010, 298(6):G985-G993.

24. Bleich S, Degner D, Wiltfang J, Maler J, Niedmann P, Cohrs S, Mangholz A, Porzig J, Sprung R, Rüther E: Elevated homocysteine levels in alcohol withdrawal. Alcohol Alcohol 2000, 35(4):351-354.

25. Stickel F, Choi SW, Kim Yl, Bagley PJ, Seitz HK, Russell RM, Selhub J, Mason JB: Effect of chronic alcohol consumption on total plasma homocysteine level in rats. Alcohol Clin Exp Res 2000, 24(3):259-264.

26. Lahiri DK, Maloney B, Zawia NH: The LEARn model: an epigenetic explanation for idiopathic neurobiological diseases. Mol Psychiatry 2009, 14(11):992-1003.

27. Lahiri DK, Maloney B: The "LEARn" (Latent Early-life Associated Regulation) model integrates environmental risk factors and the developmental basis of Alzheimer's disease, and proposes remedial steps. Exp Gerontol 2010, 45(4):291-296.

28. Beaudin AE, Stover PJ: Folate-mediated one-carbon metabolism and neural tube defects: Balancing genome synthesis and gene expression. Birth Defects Research Part C Embryo Today Reviews 2007, 81(3):183-203.

29. Bajpai R, Chen DA, Rada-Iglesias A, Zhang J, Xiong Y, Helms J, Chang CP, Zhao Y, Swigut T, Wysocka J: CHD7 cooperates with PBAF to control multipotent neural crest formation. Nature 2010, 463(7283):958-962.

30. Huang RFS, Hsu YC, Lin HL, Yang FL: Folate depletion and elevated plasma homocysteine promote oxidative stress in rat livers. J Nutr Health 2001, 131(1):33-38

31. van der Dijs FPL, Schnog JJB, Brouwer D, Velvis HJR, van den Berg GA, Bakker AJ, Duits AJ, Muskiet FD, Muskiet FAJ: Elevated homocysteine levels indicate suboptimal folate status in pediatric sickle cell patients. Am J Hematol 1998, 59(3):192-198.

32. Jacques PF, Selhub J, Bostom AG, Wilson PWF, Rosenberg $\mathbb{H}$ : The effect of folic acid fortification on plasma folate and total homocysteine concentrations. N Engl J Med 1999, 340(19):1449-1454.

33. Brauer $P$, Tierney B: Consequences of elevated homocysteine during embryonic development and possible modes of action. Curr Pharm Des 2004, 10(22):2719-2732.

34. Steegers-Theunissen RPM, Boers GHJ, Trijbels FJM, Finkelstein JD, Blom HJ, Thomas CMG, Borm GF, Wouters MGAJ, Eskes TKAB: Maternal hyperhomocysteinemia: a risk factor for neural-tube defects? Metabolism 1994, 43(12):1475-1480.

35. Kapusta L, Haagmans MLM, Steegers EAP, Cuypers MHM, Blom HJ, Eskes TKAB: Congenital heart defects and maternal derangement of homocysteine metabolism. J Pediatr 1999, 135(6):773-774.

36. Verkleij-Hagoort A, Bliek J, Sayed-Tabatabaei F, Ursem N, Steegers E, Steegers-Theunissen R: Hyperhomocysteinemia and MTHFR polymorphisms in association with orofacial clefts and congenital heart defects: $A$ meta-analysis. Am J Med Genet A 2007, 143(9):952-960.

37. Da Lee R, An SM, Kim SS, Rhee GS, Kwack SJ, Seok JH, Chae SY, Park CH, Choi YW, Kim HS: Neurotoxic effects of alcohol and acetaldehyde during embryonic development. J Toxic Environ Health A 2005, 68(23-24):2147-2162.

38. Bilotta J, Barnett JA, Hancock L, Saszik S: Ethanol exposure alters zebrafish development: a novel model of fetal alcohol syndrome. Neurotoxicol Teratol 2004, 26(6):737-743.

39. Minana R, Climent E, Barettino D, Segui J, Renau-Piqueras J, Guerri C: Alcohol exposure alters the expression pattern of neural cell adhesion molecules during brain development. J Neurochem 2000, 75(3):954-964.

40. Zhou F, Sari Y, Zhang J, Goodlett C, Li TK: Prenatal alcohol exposure retards the migration and development of serotonin neurons in fetal C57BL mice. Dev Brain Res 2001, 126(2):147-155.

41. Li YX, Yang HT, Zdanowicz M, Sicklick JK, Qi Y, Camp TJ, Diehl AM: Fetal alcohol exposure impairs Hedgehog cholesterol modification and signaling. Lab Investig 2007, 87(3):231-240.

42. Shi Y, Zhao S, Li J, Mao B: Islet-1 is required for ventral neuron survival in Xenopus. Biochem Biophys Res Commun 2009, 388(3):506-510.
43. Kashef J, Köhler A, Kuriyama S, Alfandari D, Mayor R, Wedlich D: Cadherin-11 regulates protrusive activity in Xenopus cranial neural crest cells upstream of Trio and the small GTPases. Genes Dev 2009, 23(12):1393-1398.

44. Aybar MJ, Nieto MA, Mayor R: Snail precedes slug in the genetic cascade required for the specification and migration of the Xenopus neural crest. Development 2003, 130(3):483-494.

doi:10.1186/s13041-014-0067-9

Cite this article as: Shi et al:: 5-mehtyltetrahydrofolate rescues alcohol-induced neural crest cell migration abnormalities. Molecular Brain 2014 7:67.

\section{Submit your next manuscript to BioMed Central and take full advantage of:}

- Convenient online submission

- Thorough peer review

- No space constraints or color figure charges

- Immediate publication on acceptance

- Inclusion in PubMed, CAS, Scopus and Google Scholar

- Research which is freely available for redistribution

Submit your manuscript at www.biomedcentral.com/submit
C Biomed Central 